\title{
Synthesis and evaluation of methionine and folate co-decorated chitosan self-assembly polymeric micelles as a potential hydrophobic drug-delivery system
}

\author{
CHEN YuQi ${ }^{1}$, CAO Jie ${ }^{1}$, ZHU Hong Yan $^{1}$, CUI SiSi ${ }^{1}$, WANG AQin ${ }^{1}$, QIAN ZhiYu ${ }^{2}$ \& \\ GU YueQing ${ }^{*}$ \\ ${ }^{1}$ Department of Biomedical Engineering, School of Life Science and Technology, China Pharmaceutical University, Nanjing 210009, China; \\ ${ }^{2}$ Department of Biomedical Engineering, School of Automation, Nanjing University of Aeronautics and Astronautics, Nanjing 210016, China
}

Received December 20, 2012; accepted January 8, 2013; published online March 6, 2013

\begin{abstract}
In this study, an amphiphilic copolymer folate-succinyl-methionine-chitosan-octyl (FSMCO) was successfully synthesized step by step for self-assembling polymeric micelles. The copolymers formed micelle-like nanoparticles by their amphiphilic characteristics and structures were examined by UV-Vis absorption and Fourier transform spectroscopy. The sizes of blank and ICG derivativeloaded micelles measured by dynamic light scattering were about 170 and $140 \mathrm{~nm}$, respectively, which were spherical in shape with an average zeta potential of $-10 \mathrm{mV}$. Further studies on the stability showed that the micellar solutions maintain their sizes at room temperature for 1 month without distinct aggregation or dissociation. ICG derivative was much better photostable after being entrapped by the new carrier. The prepared FSMCO micelles displayed a good drug loading content (11.7\%), entrapment efficiency (66.5\%) and sustained release rate for the model drug fluorescein. The copolymers demonstrated weeny cytotoxicity toward Bel-7402, L02 and A549 cells when incubated for $2 \mathrm{~d}$. Ligands modified micelles endowed preferable cell targeting capability and beautiful cell inhibition of HCPT-FSMCO on Bel-7402 tumor cells. This kind of polymeric micelles may be a promising nanovehicle in delivering near-infrared dyes for tumors imaging and chemotherapeutic drugs for cancer therapeutics.
\end{abstract}

folate, methionine, chitosan, micelles, ICG derivative

Citation: Chen Y Q, Cao J, Zhu H Y, et al. Synthesis and evaluation of methionine and folate co-decorated chitosan self-assembly polymeric micelles as a potential hydrophobic drug-delivery system. Chin Sci Bull, 2013, 58: 2379-2386, doi: 10.1007/s11434-013-5733-2

For a long time, numerous cytotoxic anticancer drugs display water-insoluble, poor tumor selectivity, toxic side effects [1] and the potential of resistance, and so on. The above limitations entail an urgent need for developing new drug formulation. Increasing interest has being paid to the design and development of drug delivery platforms for selective delivery (i.e. targeted delivery) to solid tumors, developing new non-invasive strategies for the diagnosis and treatment of cancer [2]. For instance, nanomicellar systems are promising carriers to figure out the above issues, which produced significant improvements in tumor accumulation of drugs and/or a reduction in systemic toxicity with en-

*Corresponding author (email: cpuyueqing@163.com) hanced permeation and retention (EPR) effect [3] and/or together with active targeting. Positively charged chitosan is an outstanding choice of semi-synthetic natural carrier materials can be modified to form micelles. Nowadays, folic acid (FA) has been proven to mediate favorable transcytosis of coupled nanocarriers through folate receptor (FR) [4] mediated pathway. The vast expression of FR in many cancers makes it a fascinating marker and target molecule for cancer diagnosis and therapy [5]. Some drugs conjugated with folate and imaging agents have arrived clinical test stage [6].

Methionine (Met) is sulfur containing essential amino acid for normal growth and development in mammals [7]. It plays many critical roles in mammalian metabolism such as 
methylation of DNA, protein synthesis, cell survival, and reproduction. Methionine can be transported by LAT1 and LAT2 receptors strongly expressed in many malignant cell membranes [8]. A characteristic feature had been found that many malignant cell lines need a large amount of exogenous methionine named methionine-dependence because of the metabolic defect of regenerating methionine, whereas non-cancerous cells are generally methionine independent $[9,10]$. Methionine restriction or even methionine-free diets was connected to cancer therapy [11]. However, methionine in this study was reacted to micelles severed as a targeting group.

With regards to the hydrophobicity of most therapeutic drugs and the ligands mentioned previously to enhance anticancer efficacy, we designed a new folate-succinyl-methionine-chitosan-octyl amphiphilic copolymer for the encapsulation of hydrophobic drugs in this study for the first time, in which FA and Met as the bi-model targeting moieties emerged an ingenious synergistic effect to amplify tumour targeting [12]. This dual receptors targeting would be a fresh tactic for delivering drugs effectively and specifically than single receptor targeting. Significant advances have been made in synthetic methodology, such that prepared the kind of micelles with highly controlled size, shape, surface charge and physicochemical characteristics, with FA and Met further decorated in their surface in order to improve biocompatibility and achieve active targeting. Fluorochromes, fluorescein and ICG derivative, were successfully encapsulated by FSMCO micelles. Identification, in vitro release and in vitro cytotoxicity of the polymeric micelles were also studied in detail. These results suggest that this novel FSMCO copolymer based on the polysaccharide is likely to be a promising nanocarrier for in vivo tumor imaging and anticancer drug delivery.

\section{Materials and methods}

\subsection{Materials}

Chitosan, with deacetylation degrees of $91 \%$ and average molecular weight (MW) of $100 \mathrm{kD}$ was purchased from Aoxing Biotechnology Co. Ltd. (Zhejiang, China). Folic acid (FA, folate, MW 441), 1-ethyl-3-(dimethylaminopropyl) carbodiimide hydrochloride (EDC·HCl), N,N'-dicyclohexylcarbodiimide (DCC), $N$-hydroxysuccinimide (NHS) and methyl thiazolyl tetrazolium (MTT) were all purchased from Sigma-Aldrich (St. Louis, USA). Indocyanine Green (ICG) derivative (MW 689) was prepared by our research group. L-Methionine, hydroxycamptothecine (HCPT) and fluorescein were purchased from Aladdin reagent (Shanghai, China). RPMI 1640 medium, trypsin, penicillin, calf serum, streptomycin and ethylenediaminetetraacetic acid (EDTA) were all purchased from Invitrogen-Life Technologies (Carlsbad, CA, USA). Many other reagents used in this study were all commercially obtained from Shanghai Chemical Reagent
Company (Shanghai, China) with analytical reagent grade.

Human hepatoma cell lines (Bel-7402), human lung carcinoma cell lines (A549) and immortal hepatic cell lines (L02) were all purchased from American Type Culture Collection (ATCC, Manassas, VA, USA).

\subsection{Synthesis of folate and methionine co-modified chitosan micelles}

(i) Preparation of $N^{\prime}$-methionine-chitosan- $N$-octyl (MCO). In brief, $0.51 \mathrm{~g}$ octyl aldehyde was dropwise added to $0.5 \mathrm{~g}$ chitosan dissolved in $40 \mathrm{~mL} 1 \%$ acetic acid, followed by constant stirring for $4 \mathrm{~h}$ at room temperature. Then, the resultant solution $\mathrm{pH}$ was adjusted to 4.5 . After that, $1.6 \mathrm{~mL}$ $\mathrm{NaBH}_{4}$ solution $(10 \mathrm{mg} / \mathrm{mL})$ was rather slowly added drop by drop and followed by continuous stirring for $12 \mathrm{~h}$. After conjugating octyl to the reactive amino groups of the chitosan, the product solution (chitosan-octyl, $\mathrm{CO}$ ) was filtered and neutralized with gradual addition of $5 \%(w / v) ~ N a O H$ solution, then purified by dialyzing against distilled water (molecular weight cut off (MWCO) $10 \mathrm{kD}$ ).

Then, diluted methionine was slowly dropwise added to the mixed solution (acidified to $\mathrm{pH}$ 5.0) of chitosan-octyl and excess EDC/NHS (molar ratio of Met:EDC:NHS=1:5:1), constant stirring for $8 \mathrm{~h}$ at room temperature to completely react to the remainder amino groups of chitosan. Still, a dialysis was done to purify the reaction product (Met-chitosanoctyl, MCO).

(ii) Synthesis of succinic anhydride modified $\mathrm{MCO}$ (SMCO). In order to obtain negatively charged nanoparticles, succinic anhydride dissolved in acetone $(3 \mathrm{~g} / 17 \mathrm{~mL})$ was reacted with MCO (2 g) for $36 \mathrm{~h}$. After that, the nanoparticles were washed several times with deionized water (DI water).

(iii) Preparation of folate-SMCO (FSMCO) micelles. $\gamma$ $\mathrm{COOH}$ group of FA $(25 \mathrm{mg})$ dissolved in anhydrous dimethyl sulfoxide (DMSO, $2.5 \mathrm{~mL}$ ) was activated for $4 \mathrm{~h}$ in the presence of DCC and NHS (molar ratio of FA:DCC:NHS= $1: 1.2: 2$ ) [13], followed by centrifuging and filtering to remove the side product dicyclohexylurea (DCU) precipitate, then continuous stirring in the dark for $12 \mathrm{~h}$ at room temperature to crosslink FA-NHS to a balanced amount of amino groups of SMCO, dialyzed, frozen and lyophilized.

\subsection{Characterization of FSMCO micelles}

To identify FSMCO, UV-Vis absorption spectra were used to analyze the synthesized products using UV-Vis spectrophotometer (JH 754PC, China). Successful preparations of FSMCO micelles were also confirmed by Fourier transform infrared (FTIR) spectra (Nicolet ECO 2000, USA).

\subsection{Preparation of model drug-loaded FSMCO micelles}

To figure out the maximal drug loading content, the optimum 
weight ratios (Model drugs: $\mathrm{FSMCO}=2: 1,1: 1,1: 2,1: 4$, $1: 5,1: 6)$ were investigated firstly. Model drugs with different hydrophobicities, fluorescein and ICG derivative, were dissolved in DMSO and dropwise added to FSMCO solution respectively with magnetic stirring at room temperature. Then, the resultant mixture was dialysed in dialysis tube (MWCO $10 \mathrm{kD}$ ) against DI water for $2 \mathrm{~d}$, followed by centrifuging at $5000 \mathrm{r} / \mathrm{min}$ for $10 \mathrm{~min}$ to remove unloaded model drugs, the resultant supernatant was lyophilized and dissolved in dimethyl formamide (DMF), the absorbance of the resultant solutions at the absorption peaks of the two model drugs were analyzed, respectively.

The drug encapsulation efficiency and drug loading content were measured based on the following equations [14]: encapsulation efficiency $(\mathrm{EE})=($ mass of drug encapsulated in micelles/mass of drug in feed $\times 100 \%$; drug loading content $=$ (mass of drug encapsulated in micelles/mass of drug encapsulated micelles) $\times 100 \%$.

\subsection{Particle size and zeta potential measurement}

Particle size is increasingly recognized as an important parameter in the effective delivery of therapeutic agents by particulate vehicles, as well as size distribution can have a profound effect on the drug release profile, organ targeting and the efficiency in penetrating tissues and cells [15]. In this study, the average sizes and polydispersity of blank and ICG derivative-loaded FSMCO micelles were characterized by Particle Size Analyzers (Brookhaven Instruments Corporation, Austin, Texas, USA) in phosphate-buffered saline (PBS, pH 7.4). Meanwhile, the zeta potential, a measure of the surface charge on the micelles, was determined by Zeta Potential Analyzers (Brookhaven Instruments Corporation, Austin, Texas, USA) in PBS (pH 7.4) solution.

\subsection{Micelle stability}

To study the storage stability, blank micelles and ICG derivative-loaded FSMCO micelles were suspended in PBS $(1.0 \mathrm{mg} / \mathrm{mL})$ and stored in dark place at room temperature for a month. Time-dependent change in micellar size was monitored by Particle Size Analyzers to evaluate the stability of the micelles.

\subsection{Transmission electron microscopy (TEM) of micelles}

The morphological evaluation of diluted blank FSMCO micelles and ICG derivative-loaded FSMCO micelles were performed using transmission electron microscope (TEM, Philips FEI Tecnai G2 20s-TWIN, the Netherlands) with accelerated voltage of $200 \mathrm{kV}$. Samples were prepared by dropping diluted micelles solutions on carbon-coated copper grids and allowed them to dry at room temperature. The specimens on the copper grids were avoided to be stained.

\subsection{Status study of model drug in micelles}

Fluorescein and ICG derivative were selected with the excitation and emission wavelengths 488/525 $\mathrm{nm}$ and 765/808 $\mathrm{nm}$, respectively. The former is suitable for in vitro cellular study under confocal microscopy and the latter is available in vivo targeting evaluation under near infrared imaging system.

Optical properties about absorption and fluorescence of free and incorporated ICG derivative were measured by UV-Vis spectrophotometer and S2000 spectrometer (Ocean Optics, USA), respectively. In addition, relative fluorescence intensity was measured under successive exposure to the laser $(765 \mathrm{~nm})$ for $1 \mathrm{~h}$ to evaluate the photostability of free and incorporated ICG derivative, and data was gathered every $5 \mathrm{~min}$.

\subsection{In vitro drug release study}

Fluorescein-loaded micelles and ICG derivative-loaded micelles were prepared to investigate the release profiles. In vitro drug release studies were carried out by a dialysis (MWCO $10 \mathrm{kD}$ ) using PBS (pH 7.4). Fluorescein and ICG derivative-loaded FSMCO solution was transferred into dialysis bags and dialyzed against $30.0 \mathrm{~mL}$ PBS in dark environment with constant agitation at $95 \pm 5 \mathrm{r} / \mathrm{min}$ and $37^{\circ} \mathrm{C}$, respectively. At selected time intervals, $3 \mathrm{~mL}$ dialysate was withdrawn and replaced with $3 \mathrm{~mL}$ fresh PBS to make the volume fixed at every time point during the assay. The concentration of the released model drugs was determined by UV-Vis spectrophotometer and calculated according to a previously obtained calibration curve. The release experiments were conducted in triplicate.

\subsection{In vitro cell viability studies}

The cytotoxicity of FSMCO was determined by employing MTT assay [16] against A549, L02 and MCF-7 cells. Cells were seeded into 96 -well plate and cultivated at $37^{\circ} \mathrm{C}$ for $24 \mathrm{~h}$. After that, cells were exposed to $200 \mu \mathrm{L}$ a series of concentrations of FSMCO solutions (0.0625, 0.125, 0.25, $0.5,1,2 \mathrm{mg} / \mathrm{mL}$ ) for another $48 \mathrm{~h}$. After incubation, FSMCO solutions were replaced with $180 \mu \mathrm{L}$ fresh RPMI 1640 medium and $20 \mu \mathrm{L}$ MTT solutions $(5 \mathrm{mg} / \mathrm{mL})$. Culture medium was removed after $4 \mathrm{~h}$, and $200 \mu \mathrm{L}$ DMSO was added into each well with continuously shake for $10 \mathrm{~min}$ to dissolve the formazan crystals. The absorbance at $490 \mathrm{~nm}$ was measured using a Microplate Reader (Model 500; BIORAD, USA). Thus the metabolic activities of the cells were defined [17] by the following equation:

$$
\text { Cell viability }(\%)=\mathrm{OD}_{\mathrm{a}} / \mathrm{OD}_{\mathrm{b}} \times 100 \% \text {, }
$$

where $\mathrm{OD}_{\mathrm{a}}$ was the optical density of the cells incubated with FSMCO micelles, and $\mathrm{OD}_{\mathrm{b}}$ was the optical density of the cells treated in the absence of FSMCO micelles only 
with RPMI 1640 medium.

\subsection{Targeting ability investigation of FSMCO at cellular level}

In order to find out the target properties of the micelles at cellular level, Bel-7402 and A549 cells with a density of 5x $10^{5}$ cells/well in confocal laser scanning microscopy (CLSM) culture dishes were incubated with fluorescein loaded nanoparticles. $2 \mathrm{~mL}$ culture medium containing $0.1 \mathrm{mg}$ fluorescein loaded succinyl-octyl-chitosan micelles [13] (SOC, pre-synthesized by our laboratory) or FSMCO was added to each well, medium was detached after $6 \mathrm{~h}$ incubation time and the cells were washed thrice with PBS. The fluorescence of the cells was obtained using CLSM (BX60, Olympus, Japan) under $488 \mathrm{~nm}$ excitation. Additionally, Bel-7402 cells were exposed to $2 \mathrm{~mL}$ culture medium containing free FA or Met or both as well as fluorescein-loaded nanoparticles for competition assay. Subsequent procedure was acted as the method described above.

\subsection{Inhibition effect of drug-loaded FSMCO micelles on Bel-7402 cells}

HCPT was encapsulated into FSMCO micelles to evaluate the suppression on Bel-7402 cells, cells were seeded in 96well culture dishes and cultured for $24 \mathrm{~h}$. HCPT, HCPTSOC, HCPT-FSMCO at various concentrations (from 3.125 to $100 \mu \mathrm{g} / \mathrm{mL}$ ) suspended in PBS were added to cell culture dishes and incubated for $24 \mathrm{~h}$. MTT assay was also carried out to detect the livability of Bel-7402 cells.

\section{Results and discussion}

\subsection{Synthesis and characterization of FSMCO}

FA, Met, CO, MCO and FSMCO were studied by UV-Vis detection, and the results (Figure 1) showed that FSMCO had all three maximum absorption of Met (210 nm), CO (228 nm) and characteristic absorption peaks of FA (280 and $362 \mathrm{~nm}$ ), which confirmed the successful acquisition of FSMCO.

Compared to chitosan, $1555 \mathrm{~cm}^{-1}$ appeared in the FTIR spectrum of MCO and FSMCO, which usually caused by secondary amines, illustrating part of amino groups of chitosan were substituted by Met and/or octyl (Figure 2). The new peaks at 2925 and $2855 \mathrm{~cm}^{-1}$ were attributed to aliphatic C-H stretching band of methyl groups of the alkyl chain $\left(-\left(\mathrm{CH}_{2}\right)_{7}-\mathrm{CH}_{3}\right)$ [18], implied that octaldehyde was reacted to chitosan. The peak at $1645 \mathrm{~cm}^{-1}$ was $\mathrm{C}=\mathrm{O}$ stretching band of acetyl group [19], confirmed that Met was linked to chitosan. Furthermore, the peak presented at $1700 \mathrm{~cm}^{-1}$ was the $\mathrm{C}=\mathrm{O}$ stretching vibration of carboxyl group, declared that succinic anhydride was reacted to part of the amino groups of MCO. The appearance of the peak at $1600 \mathrm{~cm}^{-1}$ in the FTIR spectrum of FSMCO was related to

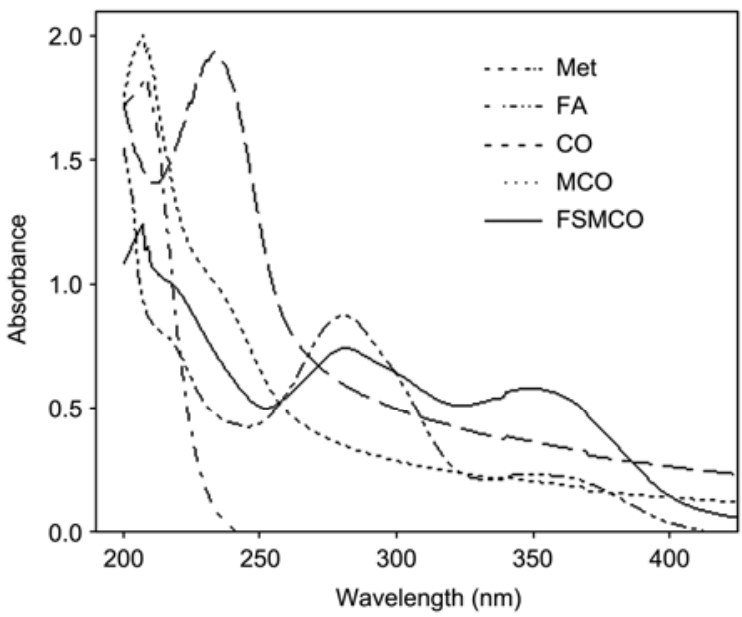

Figure 1 Identification of FSMCO with UV-Vis: absorbance of Met, FA, $\mathrm{CO}, \mathrm{MCO}$, and FSMCO.

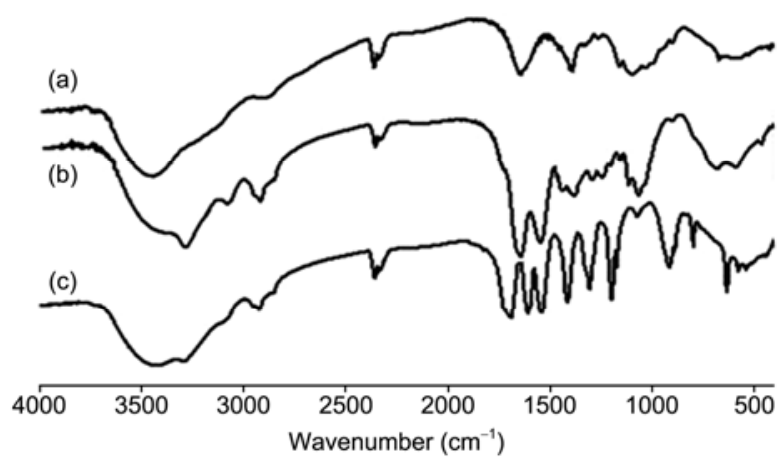

Figure 2 Identification of FSMCO with FTIR: the FTIR spectra of chitosan (a), MCO (b) and FSMCO (c).

benzene ring of the folic acid. Both the above characterizations showed that the desired copolymers were synthesized successfully.

\subsection{Size and morphology of micelles}

Studies have shown that the perfect particle size for cancer treatment in the range of 70 to $200 \mathrm{~nm}$, resulting in efficient accumulation in solid tumor tissues [20,21]. The hydrodynamic diameters of blank micelles and ICG derivativeloaded FSMCO micelles in PBS determined by dynamic light scattering (DLS) were shown in Figure 3. Protonation was avoided in PBS with $\mathrm{pH} 7.4$ [22], and the size of the blank micelles was about $170 \mathrm{~nm}$, and the size of the ICG derivative-loaded micelles was about $140 \mathrm{~nm}$, which suggested that the micelle nanoparticles had satisfactory dimensions.

The TEM micrograph (Figure 4) clearly revealed that the blank micelles and ICG derivative-loaded micelles were spherical in shape, which had a homogeneous size distribution (Polydispersity $<0.2$ ) with an average size of 140 and $110 \mathrm{~nm}$, respectively. When encapsulate with hydrophobic 

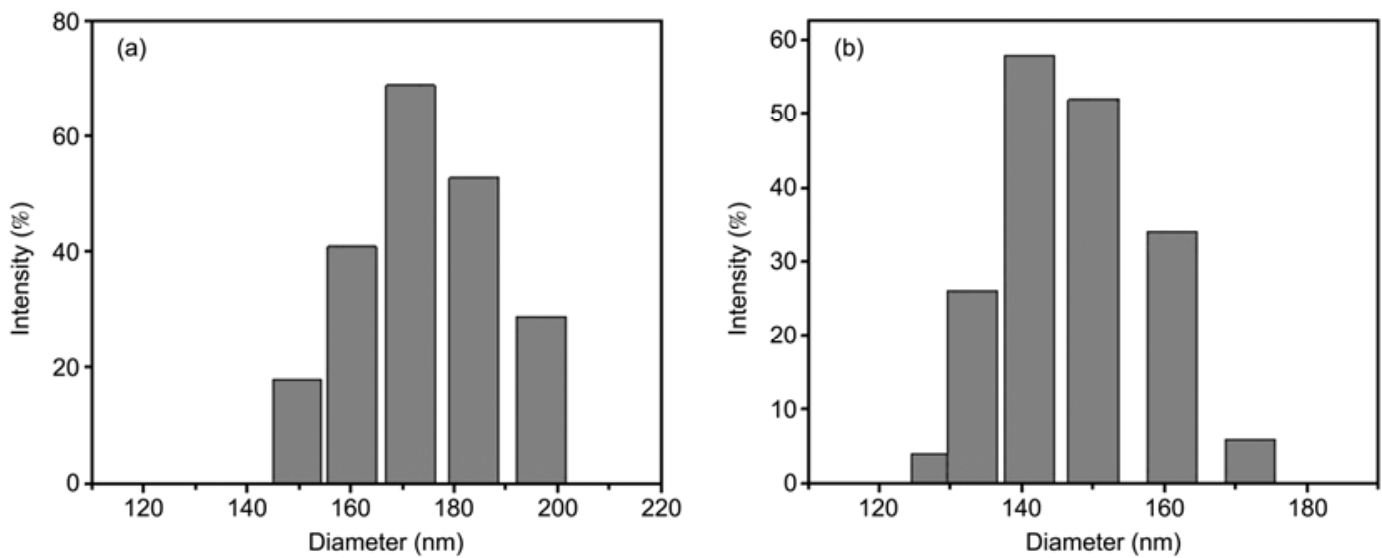

Figure 3 Size distributions of blank FSMCO micelles (a), and ICG derivative-loaded FSMCO micelles (b) at $25^{\circ} \mathrm{C}$ in PBS (pH 7.4).

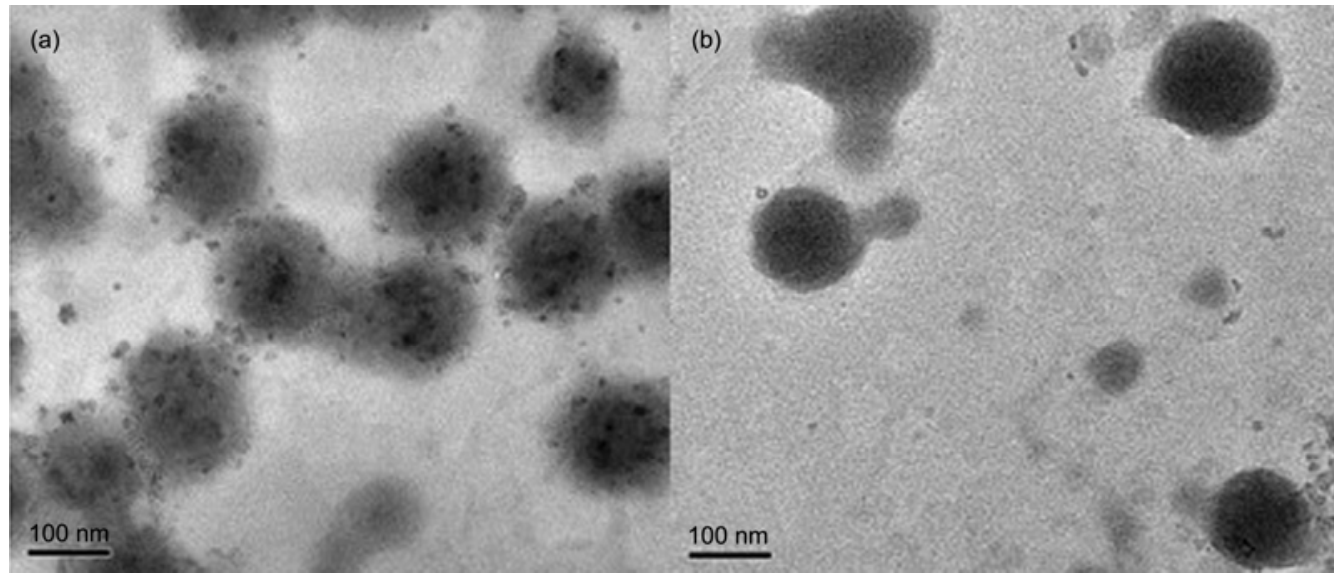

Figure 4 Transmission electron microscope (TEM) pictures of blank FSMCO (a), and ICG derivative-loaded FSMCO micelles (b).

dye ICG derivative, the hydrophobic interaction between ICG derivative and octyl would bring about the hydrophobic core much more intense, representing the decreased size compared to blank micelles; one ICG derivative molecule possesses two "-COO-", repulsive force increased among micelles because of the negative charge of the entirety increased after encapsulating with ICG derivative, thus acquired better dispersibility tested by Particle Size Analyzers. The smaller sizes were observed by TEM as compared to that determined by DLS is most likely due to the evaporation of water around the micelles during the sample preparation of TEM, which caused the shrinkage of the hydrophilic shell [23].

The zeta potential gives an indication in the physical stability of the colloidal system in vitro and in vivo, and affects its interaction with proteins and cell membranes in blood vessels [24]. MCO was positively charged, and blank micelles and ICG derivative-loaded micelles were both negatively charged about $-10 \mathrm{mV}$. It was proved that succinic anhydride was successfully reacted to MCO. Thereby, negatively charged colloidal system would barely adhere to cells in vessels when intravenous injection.

\subsection{Stability of micelles}

Diluted blank micelles solution and ICG derivative-loaded micelles solution were used in the stability study. The results (Figure 5) indicated that the micelles were almost

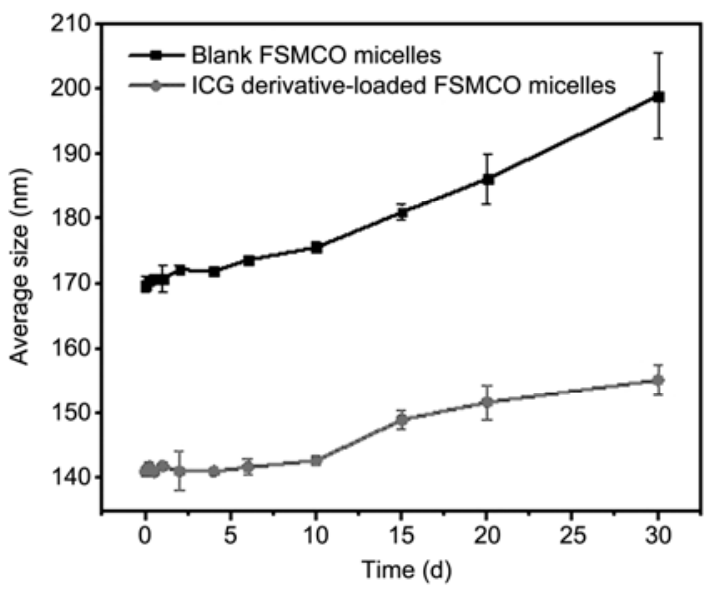

Figure 5 Particle size of blank FSMCO micelles, and ICG derivativeloaded FSMCO micelles at $25^{\circ} \mathrm{C}$ in PBS (pH 7.4) as a function of time. 
steady during storage at room temperature $\left(25^{\circ} \mathrm{C}\right)$ for a month. No change in the micelle size and size distribution in the first $10 \mathrm{~d}$ and no precipitation of ICG derivative were found during the month, moreover, ICG derivative-loaded micelles were more stable than blank micelles because of the similar polarity between octyl and ICG derivative which enhanced the hydrophobic association action.

\subsection{Drug loading and in vitro drug release}

We concluded the maximum drug loading content with the feed ratio 1:5. The optimal drug loading content was $11.74 \%$ for fluorescein (EE=66.52\%), while the drug loading content was just $37.46 \%$ for ICG derivative ( $\mathrm{EE}=6.97 \%$ ). The drug loading content and $\mathrm{EE}$ for fluorescein were more than that of the more hydrophobic ICG derivative which may be due to the rapid precipitate of the intense hydrophobicity when at the dynamic drug loading process in aqueous solution. Consequently, precipitation resulted in lower entrapment into the inner hydrophobic core.

Figure 6 shows in vitro release profile of model drugs from FSMCO micelles at $37^{\circ} \mathrm{C}$ in PBS solution ( $\mathrm{pH}$ 7.4). Obvious burst release was observed in the release profile of fluorescein and the cumulative percentage release reached about $70 \%$ during the initial $40 \mathrm{~h}$. More than $90 \%$ of the loaded fluorescein was released in the following slow release stage for up to $96 \mathrm{~h}$. In contrast to fluorescein, the ICG derivative cumulative percentage release by $240 \mathrm{~h}$ reached only $59 \%$ with a continuous slow-release started from about the 5th hour. The results were manifest and indicated that the release rate of model drug was closely related to the hydrophobicity, the hydrophobic binding force of fluorescein to the inner octyl of micelles was much weaker than that of ICG derivative because of its inferior hydrophobicity, which induced a fairly fast release rate for fluorescein from

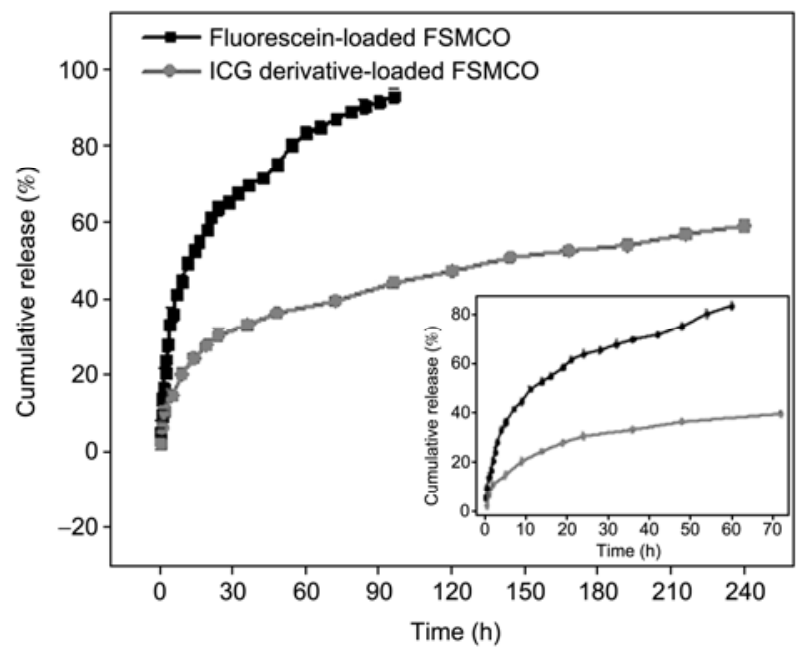

Figure 6 Release kinetics of fluorescein-loaded FSMCO micelles and ICG derivative-loaded FSMCO micelles in PBS ( $\left.\mathrm{pH} 7.4,37^{\circ} \mathrm{C}\right)$. Inset: The release kinetics of the initial $72 \mathrm{~h}$. Data were given as mean $\pm \operatorname{SD}(n=3)$.

\section{FSMCO carrier.}

\subsection{Optical characterization of entrapped ICG derivative}

ICG derivative was easily packaged into FSMCO by hydrophobic association effect. The excitation and emission spectra for ICG derivative and ICG derivative-loaded FSMCO are shown in Figure 7(a) and (b). No change was observed
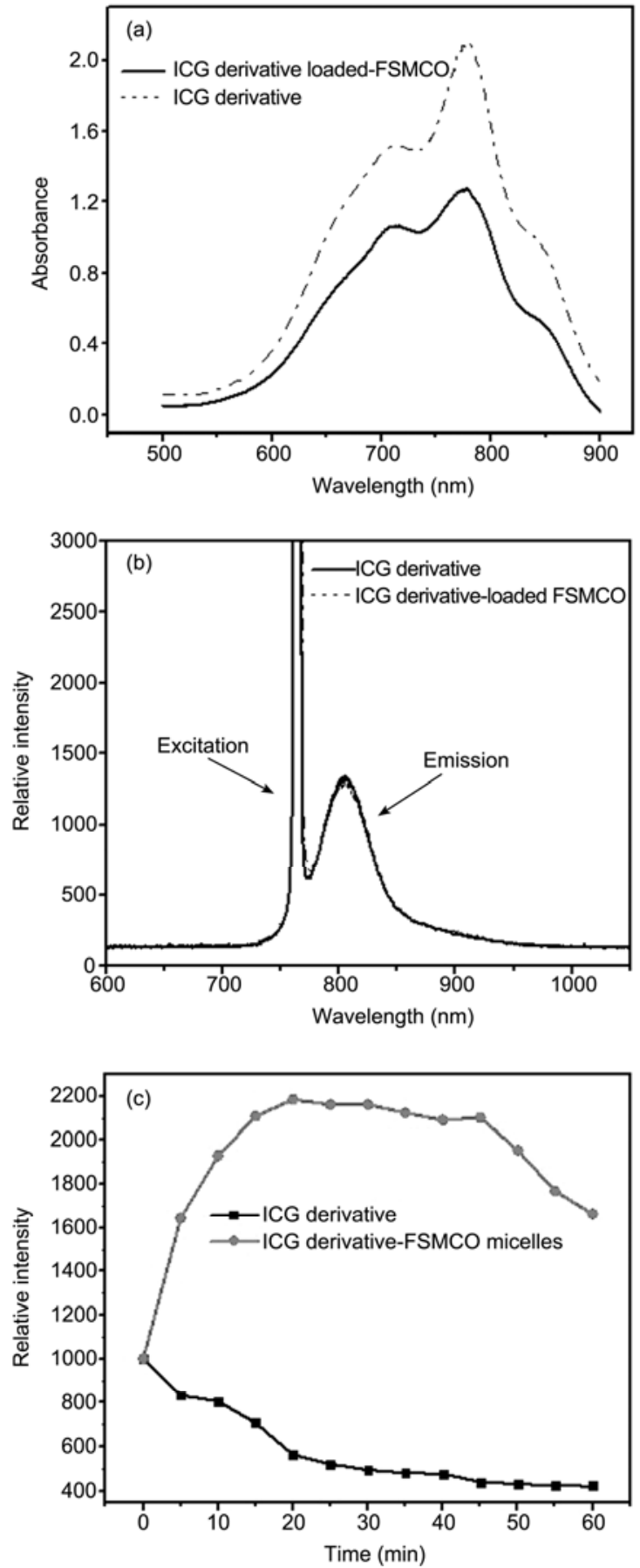

Figure 7 Optical characterization of free ICG derivative and ICG derivativeloaded FSMCO. (a) Absorbance spectra; (b) fluorescence spectra; (c) light stability. 
in optical properties of ICG derivative-loaded micelles compared to free ICG derivative, including maximum absorption peak, excitation wavelength and emission wavelength. Furthermore, better resistance to fluorescent bleaching was found after ICG derivative being encapsulated into FSMCO micelles (Figure 7(c)), the relative intensity of fluorescent emitted from ICG derivative encapsulated by FSMCO was strengthened from 1000 to nearly 2200 in the beginning 20 min, slow decrease was found in the following time, but still much higher than the relative intensity of fluorescent emitted from free ICG derivative. It was suggested that ICG derivative together with FSMCO micelles can be used to trace the dynamic process of FSMCO in the mouse model evaluated by near-infrared imaging system [25].

\subsection{Cytotoxicity assay of FSMCO micelles}

The in vitro cytotoxicities of prepared FSMCO against Bel7402, L02 and A549 cells are shown in Figure 8. The composite was proved to be weeny cytotoxicity even in $2 \mathrm{mg} / \mathrm{mL}$ by MTT assay. After the $48 \mathrm{~h}$ incubation, cell viabilities in the presence of micelles at different concentrations from 0.0625 to $2 \mathrm{mg} / \mathrm{mL}$ were more than $93 \%$, even at high con-

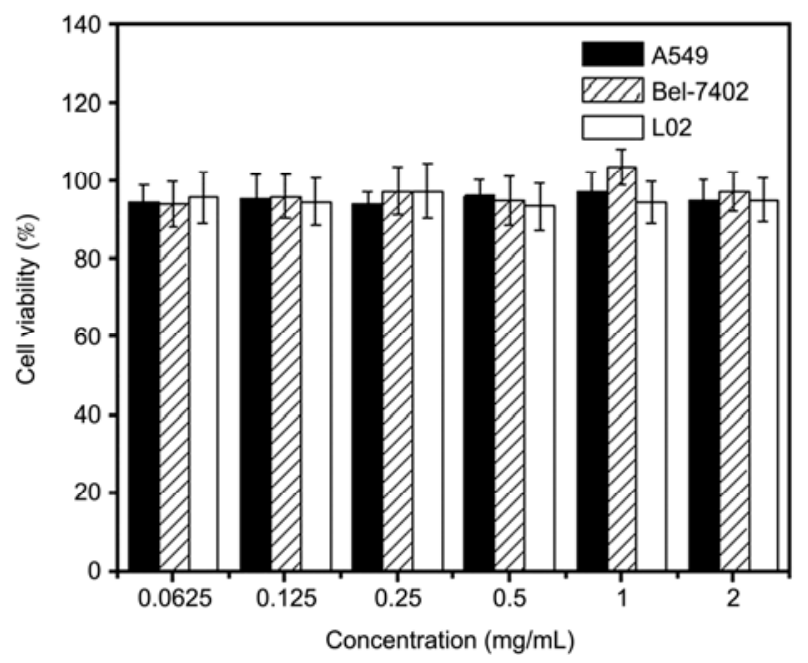

Figure 8 Cytotoxicity study of FSMCO micelles against A549 cells, Bel-7402 cells, and L02 cells. Data represent mean $\pm \operatorname{SD}(n=5)$. centration. It was therefore presupposed that the micelles exhibited reasonable biocompatibility. The confirmation of little or nontoxicity of chitosan-based micelles was also reported in other places $[26,27]$.

\subsection{Cellular uptake of nanoparticals}

The exceeded expression of LAT1, LAT2 and FA receptors of Bel-7402 cells was previously reported, while the expression levels of both receptors on A549 cells (used as control) were low $[13,28]$. In order to prove the increased uptake in tumor cells, results (Figure 9) were observed by fluorescence microscope. Fluorescence intensity of fluorescein in cells was decreased as the following order: FSMCOfluorescein $>$ FSMCO-fluorescein together with Met>FSMCOfluorescein together with FA>FSMCO-fluorescein together with FA and Met>SOC-fluorescein. Extra added free FA and Met in medium reduced the endocytosis because of the competitive inhibition. By contrast, low fluorescence intensity can be seen in A549 cells visibly. These results indicated that both the presence of FA and Met modified on micelles can promote cellular uptake effectively by Bel-7402 cells.

\subsection{Inhibition effect of HCPT loaded FSMCO micelles on celluar level}

The results (Figure 10) of MTT assay confirmed that the antitumor efficacy was advanced as the concentration of drug increased. After $24 \mathrm{~h}$ incubation, therapeutic effects were shown to varying degrees: HCPT-FSMCO $>$ HCPT $>$ HCPTSOC. HCPT-FSMCO put up greatest cell inhibition. Cell viability was down to $46 \%$ at the maximum concentration of HCPT-FSMCO due to the receptors-mediated endocytosis. Results here demonstrated that folic acid and methionine played indispensable roles in increasing the cytotoxic effect of HCPT.

\section{Conclusions}

The newly developed folate-succinyl-methionine-chitosanoctyl amphiphilic copolymer in this paper could self-assemble

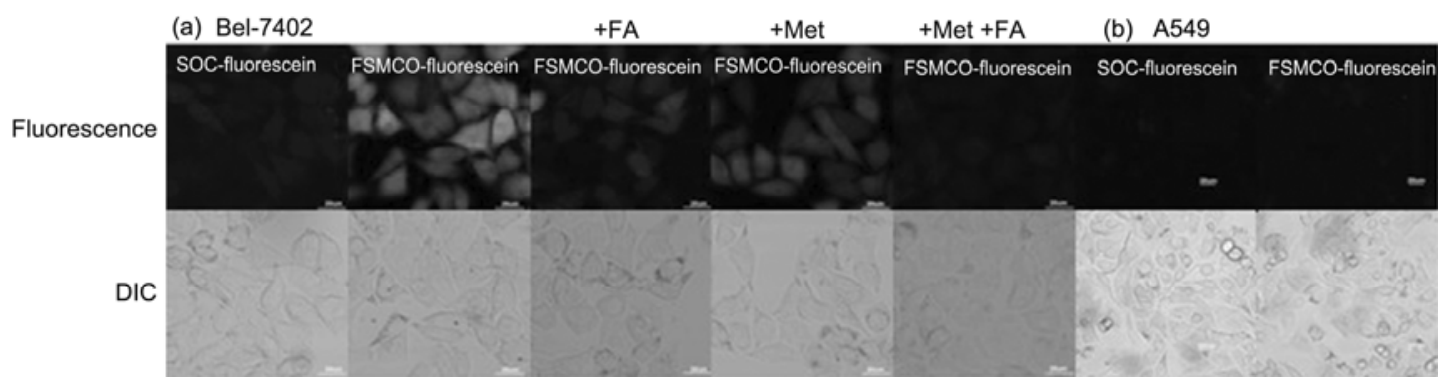

Figure 9 The fluorescence microscopy images of Bel-7402 cells (a) and A549 (b) tumor cells after incubated with fluorescein loaded micelles (scales in images were $20 \mu \mathrm{m})$. 


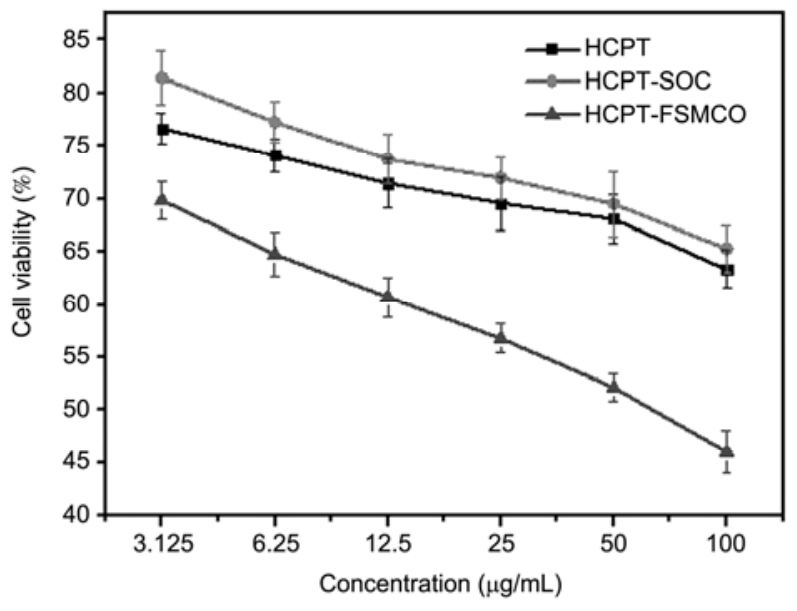

Figure 10 Anti-tumor capacity assessment of HCPT-FSMCO, HCPTSOC, HCPT in Bel-7402 tumor cells. Cell viability (\%) at $24 \mathrm{~h}$.

into core-shell micelles in aqueous medium by hydrophobic interactions, which proved to be an excellent water-insoluble drug reservoir due to the octyl as the hydrophobic core. FSMCO micelles may be used for drug delivery of hydrophobic anti-cancer drugs without any influence to drugs, which showed enhanced stability indeed. The obtained nanomicelles had spherical shape with uniform negatively charged particle size, more cyto-compatible with cancer cells or normal cells. Fluorescein encapsulation efficiency as high as $66.5 \%$ was achieved and drug loading reached to $11.7 \%$. Dual-receptor meditated selective Bel-7402 cells targeting capability and preferable cell inhibition efficacy were verified. These particular properties of FSMCO conjugates demonstrated that it is a promising injectable nanosystem for drug delivery and imaging in cancer research.

This work was supported by the National Natural Science Foundation of China (81071194) and the Graduate Innovation Project of Jiangsu Province (CXLX11_0795).

1 Werner M E, Karve S, Sukumar R, et al. Folate-targeted nanoparticle delivery of chemo- and radiotherapeutics for the treatment of ovarian cancer peritoneal metastasis. Biomaterials, 2011, 32: 8548-8554

2 Barreto J A, Malley W O, Kubeil M, et al. Nanomaterials: Applications in cancer imaging and therapy. Adv Healthcare Mater, 2011, 23: $18-40$

3 Maeda H, Bharate G Y, Daruwalla J. Polymeric drugs for efficient tumor-targeted drug delivery based on EPR-effect. Eng Polymers Control Drug Delivery Target, 2009, 71: 409-419

4 Low P S, Kularatne S A. Folate-targeted therapeutic and imaging agents for cancer. Curr Opin Chem Biol, 2009, 13: 256-262

5 Low P S, Henne W A, Doorneweerd D D. Discovery and development of folic-acid-based receptor targeting for imaging and therapy of cancer and inflammatory diseases. Acc Chem Res, 2008, 41: 120-129

6 Xia W, Low P S. Folate-targeted therapies for cancer. J Med Chem, 2010, 53: 6811-6824

7 Finkelstein J D. Methionine metabolism in mammals. J Nutr Biochem, 1990, 1: 228-237
8 Lin J, Raoof D A, Thomas D G, et al. L-type amino acid transporter-1 overexpression and melphalan sensitivity in Barrett's adenocarcinoma. Neoplasia, 2004, 6: 74-84

9 Halpern B C, Clark B R, Hardy D N, et al. The effect of replacement of methionine by homocystine on survival of malignant and normal adult mammalian cells in culture. Proc Natl Acad Sci USA, 1974, 71: 1133-1136

10 Cavuoto P, Fenech M F. A review of methionine dependency and the role of methionine restriction in cancer growth control and life-span extension. Cancer Treatment Rev, 2012, 38: 726-736

11 Pavillard V, Nicolaou A, Double J A, et al. Methionine dependence of tumours: A biochemical strategy for optimizing paclitaxel chemosensitivity in vitro. Biochem Pharmacol, 2006, 71: 772-778

12 Maltzahn G V, Park J H, Lin K Y, et al. Nanoparticles that communicate in vivo to amplify tumour targeting. Nat Mater, 2011, 10: 545-551

13 Zhu H Y, Liu F, Guo J, et al. Folate-modified chitosan micelles with enhanced tumor targeting evaluated by near infrared imaging system. Carbohydrate Polymers, 2011, 86: 1118-1129

14 Wang X H, Tian Q, Wang W, et al. In vitro evaluation of polymeric micelles based on hydrophobically-modified sulfated chitosan as a carrier of doxorubicin. J Mater Sci Mater Med, 2012, 23: 1663-1674

15 Al Kobiasi M, Chua B Y, Tonkin D, et al. Control of size dispersity of chitosan biopolymer microparticles and nanoparticles to influence vaccine trafficking and cell uptake. J Biomed Mater Res Part A, 2012, 100A: 1859-1867

16 Mosmann T. Rapid colorimetric assay for cellular growth and survival: Application to proliferation and cytotoxicity assays. J Immunol Methods, 1983, 65: 55-63

17 Lian H, Sun J, Yu Y P, et al. Supramolecular micellar nanoaggregates based on a novel chitosan/vitamin E succinate copolymer for paclitaxel selective delivery. Inter J Nanomed, 2011, 6: 3323-3334

18 Luo Y L, Yao X J, Yuan J F, et al. Preparation and drug controlledrelease of polyion complex micelles as drug delivery systems. Colloids and Surfaces B: Biointerfaces, 2009, 68: 218-224

19 Quinnones J P, Gothelf K V, Kjems J, et al. Self-assembled nanoparticles of glycol chitosan-Ergocalciferol succinate conjugate, for controlled release. Carbohydrate Polymers, 2012, 88: 1373-1377

20 Gaumet M, Vargas A, Gurny R, et al. Nanoparticles for drug delivery: The need for precision in reporting particle size parameters. Eur $\mathbf{J}$ Pharm Biopharm, 2008, 69: 1-9

21 Matsumura Y, Maeda H. A new concept for macromolecular therapeutics in cancer chemotherapy: Mechanism of tumoritropic accumulation of proteins and the antitumor agent smancs. Cancer Res, 1986, 46: 6387-6392

22 Fan L, Li F, Zhang H T, et al. Co-delivery of PDTC and doxorubicin by multifunctional micellar nanoparticles to achieve active targeted drug delivery and overcome multidrug resistance. Biomaterials, 2010, 31: 5634-5642

23 Sun P J, Zhou D H, Gan Z H. Novel reduction-sensitive micelles for triggered intracellular drug release. J Contr Release, 2011, 155: 96-103

24 Chavanpatil M D, Khdair A, Panyam J. Nanoparticles for cellular drug delivery: Mechanisms and factors influencing delivery. J Nanosci Nanotechnol, 2006, 6: 2651-2663

25 Ozdemir V, Jones B W, Glatt S J, et al. Shifting emphasis from pharmacogenomics to theragnostics. Nat Biotechnol, 2006, 24: 942-946

26 Liang N, Sun S P, Li X F, et al. $\alpha$-Tocopherol succinate-modified chitosan as a micellar delivery system for paclitaxel: Preparation, characterization and in vitro/in vivo evaluations. Inter J Pharmaceutics, 2012, 423: 480-488

27 Hu B, Ting Y W, Zeng X X, et al. Cellular uptake and cytotoxicity of chitosan-caseinophosphopeptides nanocomplexes loaded with epigallocatechin gallate. Carbohydrate Polymers, 2012, 89: 362-370

28 Chen H Y, Li B W, Ren X Y, et al. Multifunctional near-infraredemitting nano-conjugates based on gold clusters for tumor imaging and therapy. Biomaterials, 2012, 33: 8461-8476

Open Access This article is distributed under the terms of the Creative Commons Attribution License which permits any use, distribution, and reproduction in any medium, provided the original author(s) and source are credited. 